Scientific Journal of Hamadan Nursing \& Midwifery Faculty - ISSN 2008-2819

\title{
The Effect of Education Based on Health Belief Model on Preventive Behaviors Towards Cardiovascular Disease
}

\author{
Nahid Mohammadi ${ }^{1}$, Nasrin Soltani², Roya Amini ${ }^{1 *}$, Leili Tapak ${ }^{3}$
}

1. Instructor, Department of Community Health Nursing, School of Nursing and Midwifery, Hamadan University of Medical Sciences, Hamadan, Iran

2. MSc. Student in Nursing, Student Research Committee, Hamadan University of Medical Sciences, Hamadan, Iran

3. Assistant Professor, Department of Biostatistics, School of Public Health, Hamadan University of Medical Sciences, Hamadan, Iran

\begin{tabular}{lll}
\hline Article Info & Abstract \\
\hline
\end{tabular}

Received: $\quad 2017 / 11 / 15$

Accepted: $\quad 2017 / 12 / 24$

Published Online 2018/03/16

DOI:

10.30699/sjhnmf.26.4.227

Original Article

Use your device to scan and read the article online

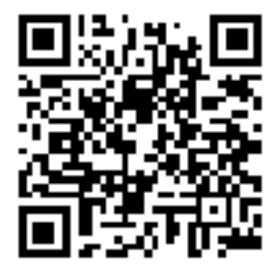

Introduction: Cardiovascular diseases are associated with individual behaviors in childhood, adolescence and youth. This article aimed to study the effect of education based on HBM on the prevention of heart disease in female students.

Methods:This pre and post-test semi-experimental study which has two groups was conducted on 126 girls of high schools in 2017. A randomized cluster sampling method and relative randomize method were used in the first and second stages respectively. At first both groups completed a questionnaire and then instructions were given to intervention group in three sessions (1 hour) on the basis of HBM. Two months later, data was collected via questionnaires in two groups again. Statistical analysis of the data was performed by SPSS software, using independent and paired t-test and Chi-square test.

Results: The two groups were similar in terms of demographic characteristics and knowledge and constructs of the health belief model at the beginning of the study and there was no significant difference in analysis $(P>0.05)$. After intervention, the mean scores of knowledge and model constructs, sensitivity, benefits, self-efficacy and behavior had significantly increased in the test group $(P<0.05)$. The perceived barriers were significantly reduced in the test group $(P<0.05)$. There were no significant differences between the perceived severity and the areas of practice according to the t-test results in both the experimental and control groups before and after the training $(P>0.05)$.

Conclusion: According to the results, education based on health belief model increases knowledge and practice in girls; so it is recommended to use this model in programs for the prevention.

Keywords: Health Belief Model, Students, Cardiovascular disease

Copyright $(C)$ 2018, Sci J Hamadan Nurs Midwifery Fac. This is an open-access article distributed under the terms of the Creative Commons Attribution-noncommercial 4.0 International License which permits copy and redistribute the material just in noncommercial usages, provided the original work is properly cited.

How to Cite This Article:

Mohammadi N, Soltani N, Amini R, Tapak L. The Effect of Education Based on Health Belief Model on Preventive Behaviors Towards Cardiovascular Disease. Sci J Hamadan Nurs Midwifery Fac. 2018; 26 (4) :227-236 
مجله علمى دانشكدة هرستارى و مامايى همدان - شايا الكترونيك: 19

مقاله يروهشى

تأثير آموزش براساس مدل اعتقاد بهداشتى بر رفتارهاى ييشغيرى كننده از بيمارىهاى قلبى

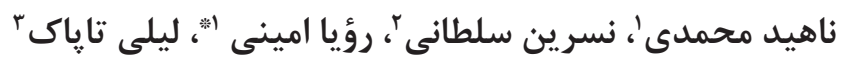

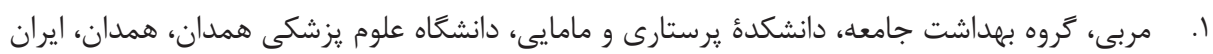

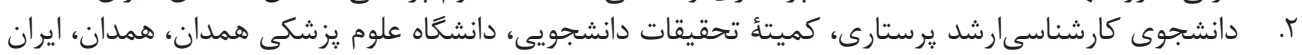

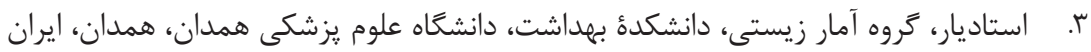

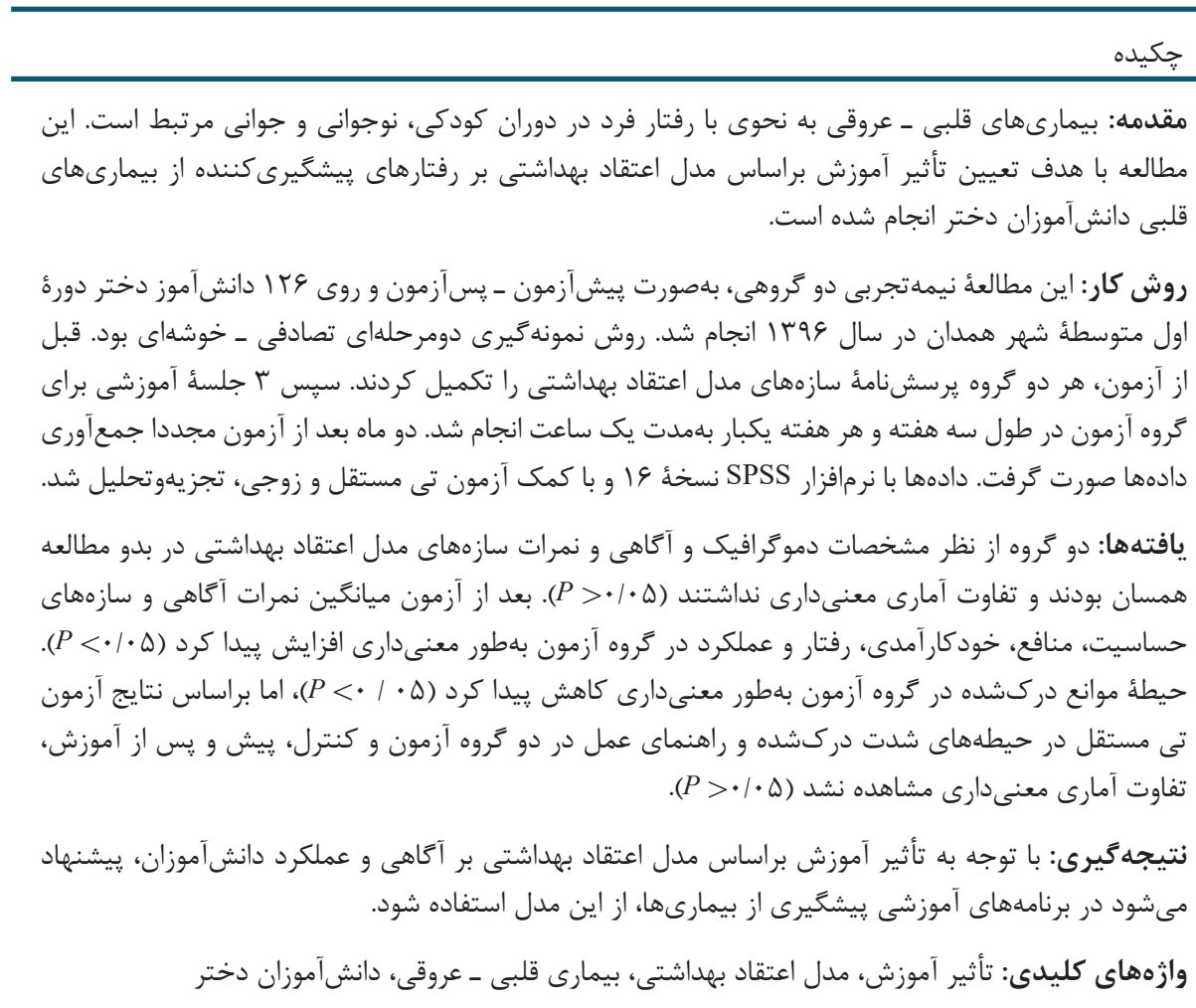

اطلاعات مقاله

تاريخ وصول: : ت ت

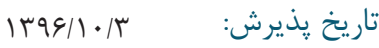

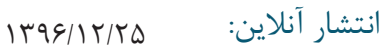

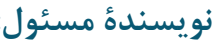

رؤيا امينى

مربى، زروه بهداشت جامعهة،

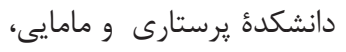

دانشعاه علوم يزشكى همدان،

همدان، ايران

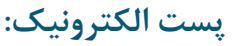

royamini@umsha.ac.ir

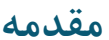

بيمارى قلبى و عروقى، بيمارى پيشروندهاى است كه از

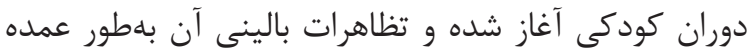

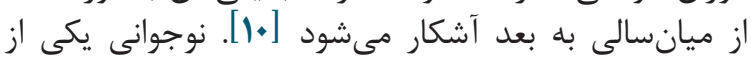

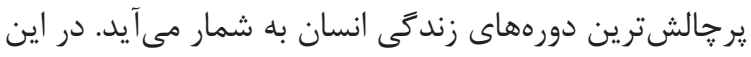

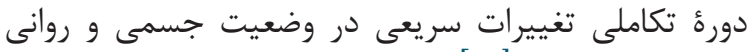

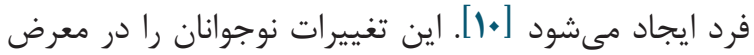

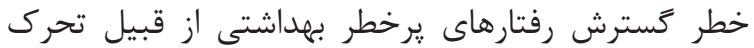

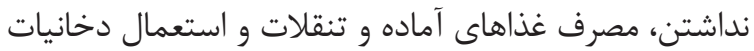

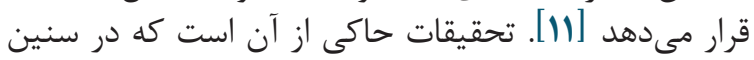

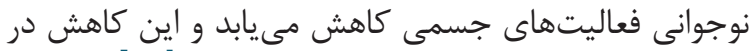

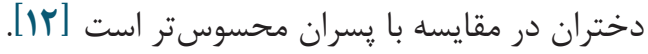

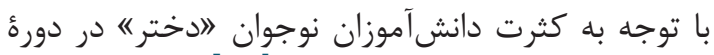

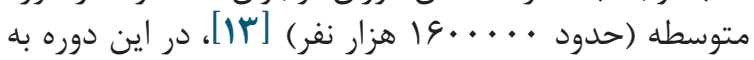

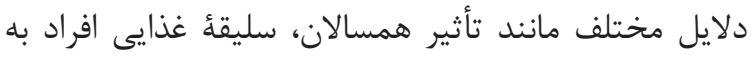

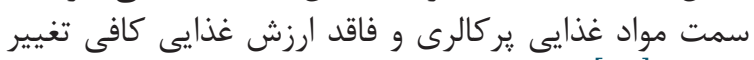

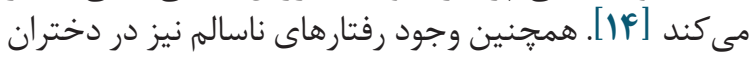

در بسيارى از كشورهاى جهان از جمله ايران، به دليل

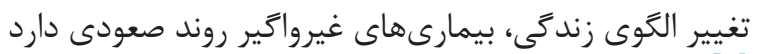

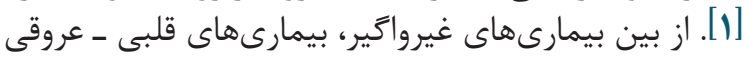

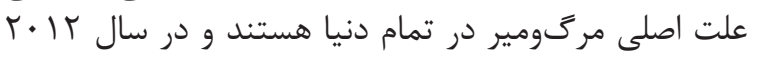

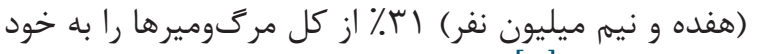

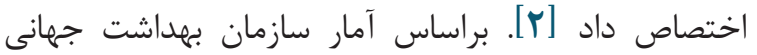
اين ميزان تا سال • WHO

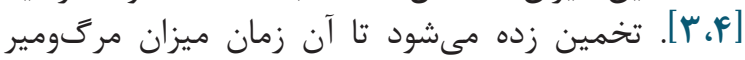

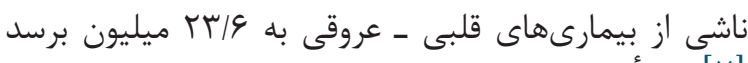

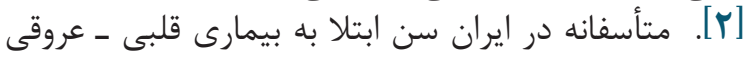

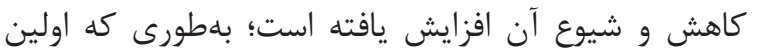

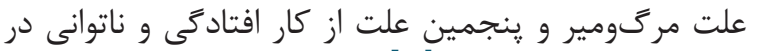

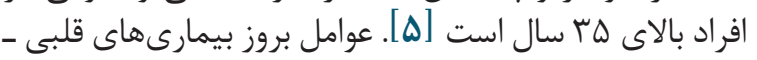

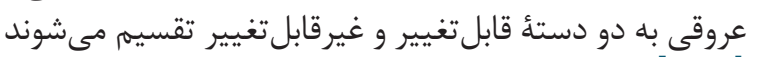

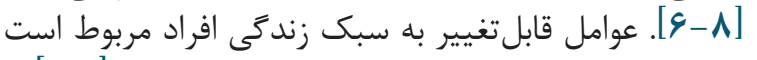

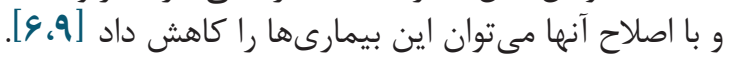




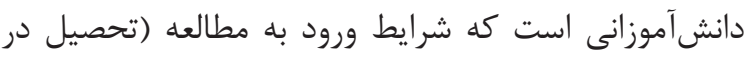

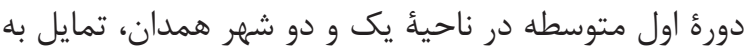

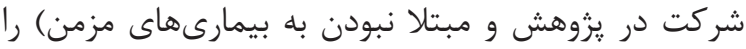

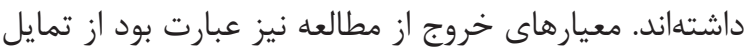

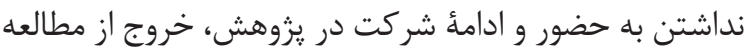

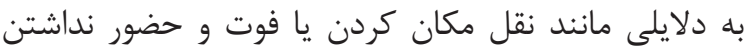

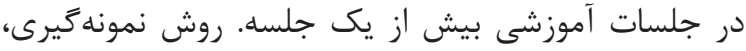

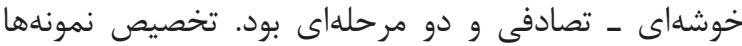

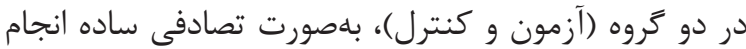

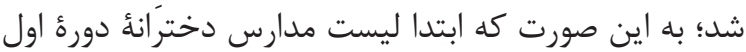

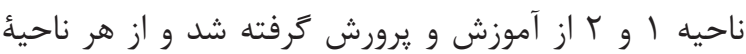

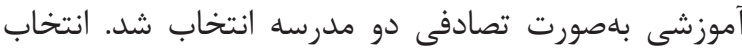

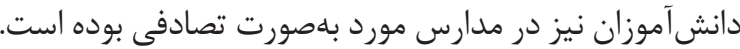

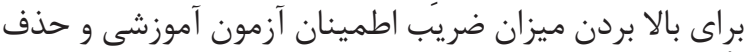

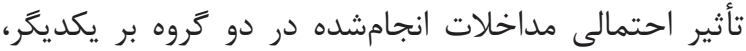

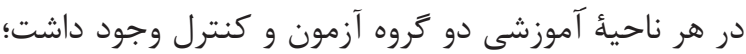

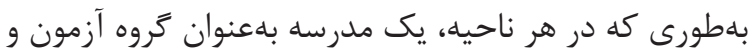

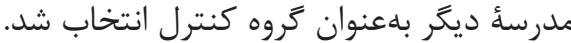

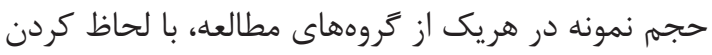

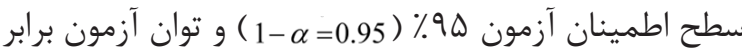

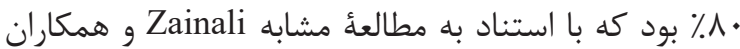

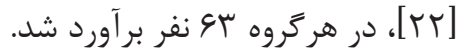

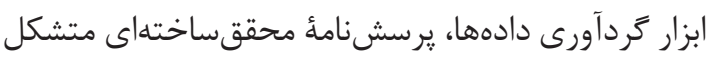

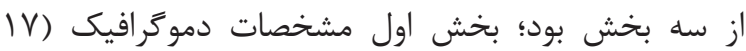

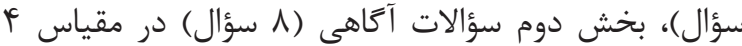

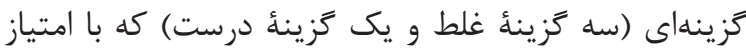

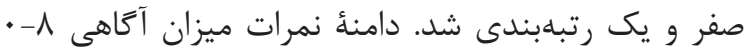

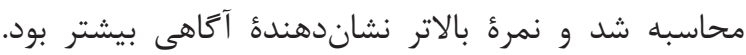

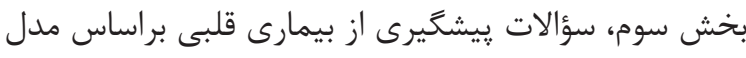

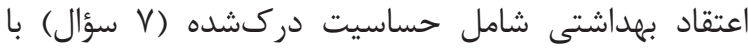

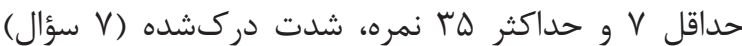

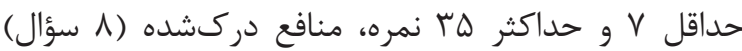

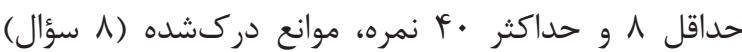

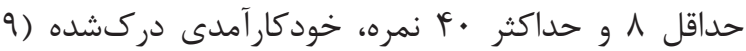

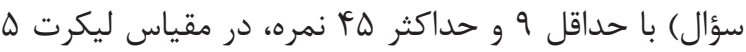

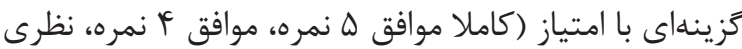

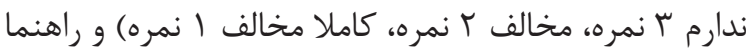

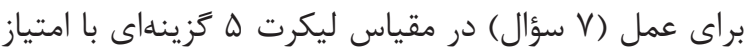

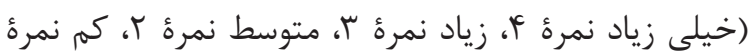

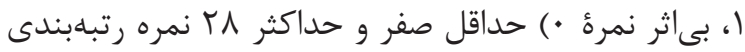

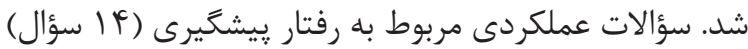

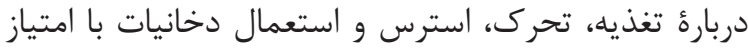

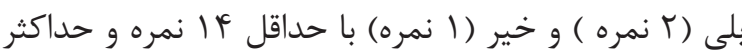

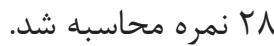

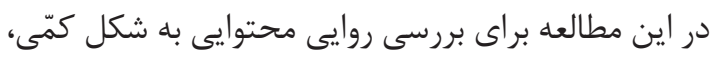

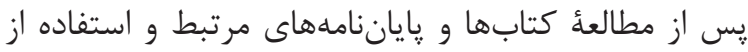

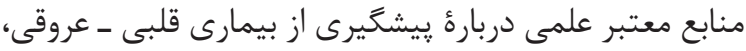

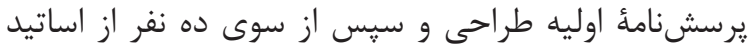

از عوامل خطرساز بيمارىهاى قلبى در سالهاى آتى بوده

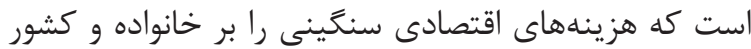

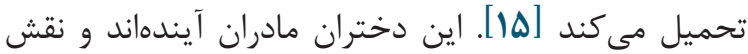

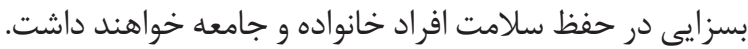

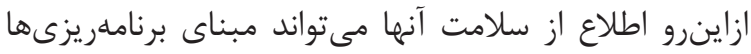

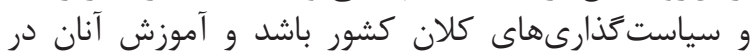

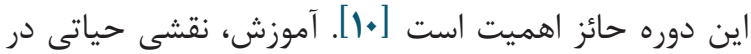

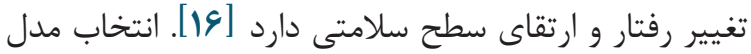

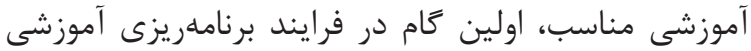

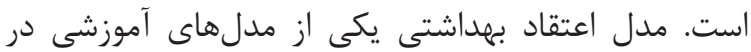

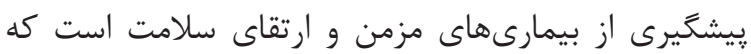

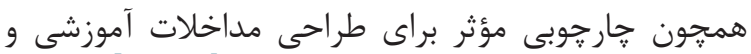

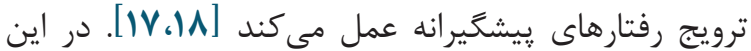

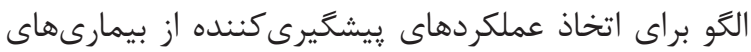

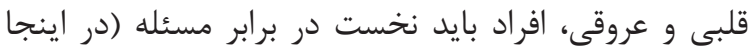

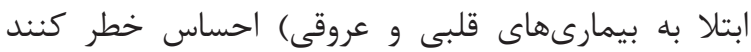

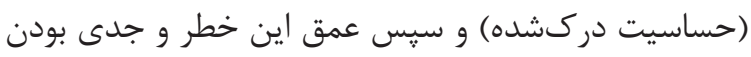

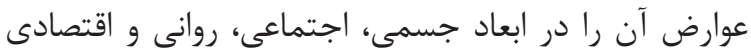

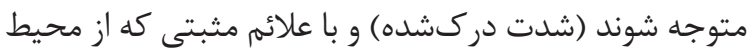

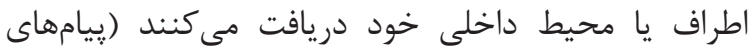

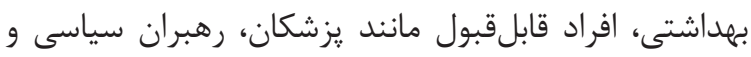

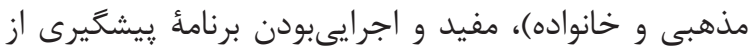

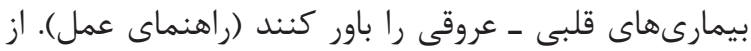

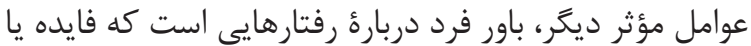

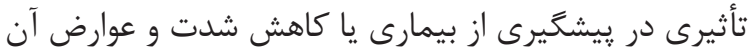

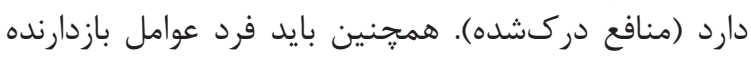

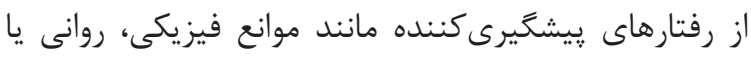

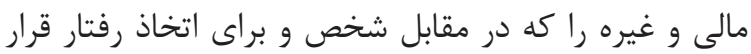

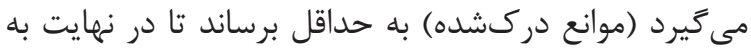

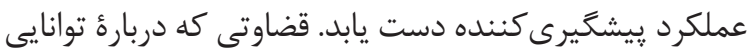

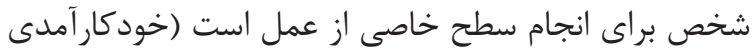

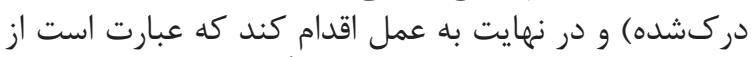

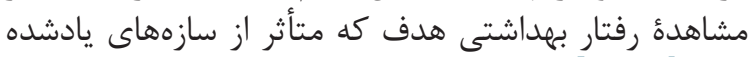

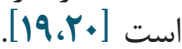

يرستاران بهعنوان عضوى كليدى، نقش مهمى در آموزي

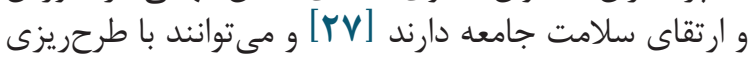

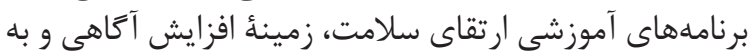

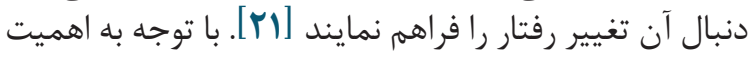

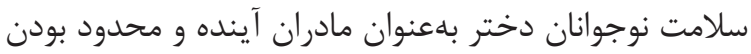

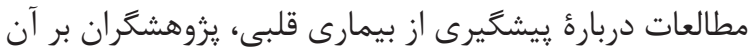

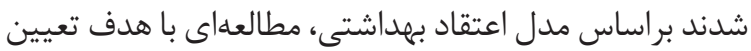

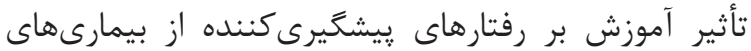

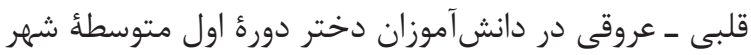

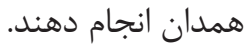

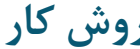

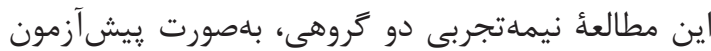

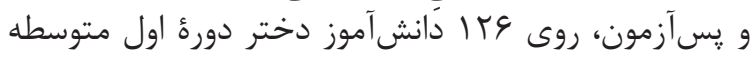

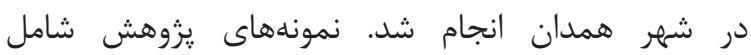


دانشرآموزان كروه كنترل، در خانواده سابقهُ ابتلا به ديابت،

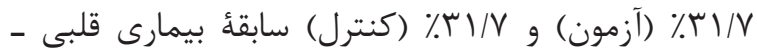

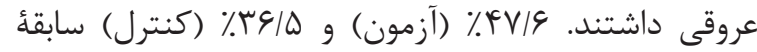

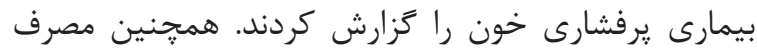

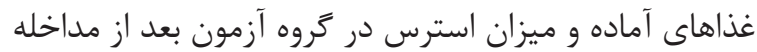
كاهش يافت.

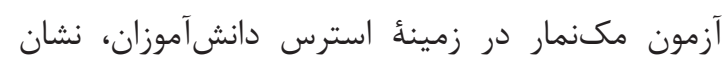

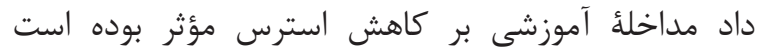

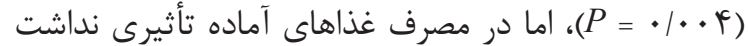

$(P=\cdot / \mathrm{V} T)$

نتايج آمارى نشان مىدهد براساس آزمون تى مستقل،

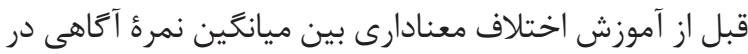

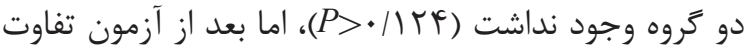

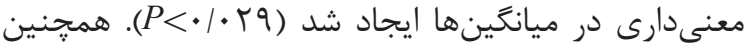

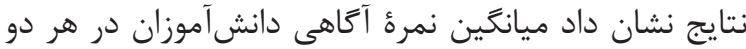

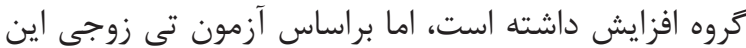

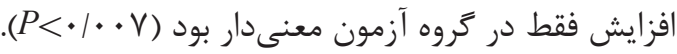

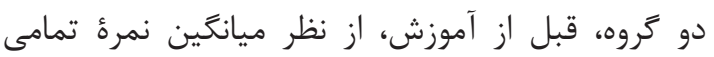

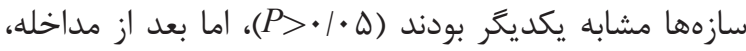

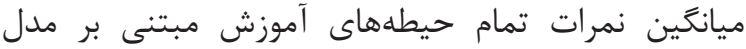

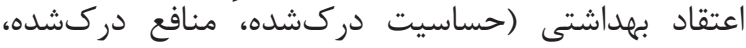

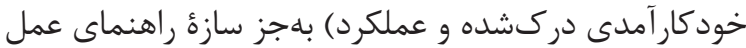

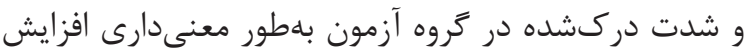

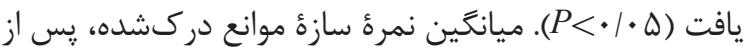

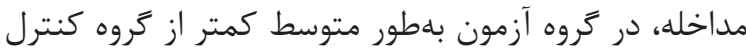

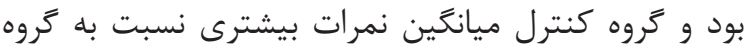

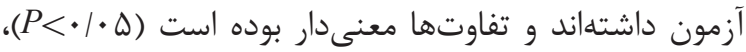

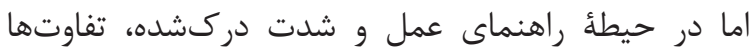

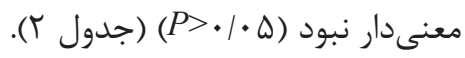

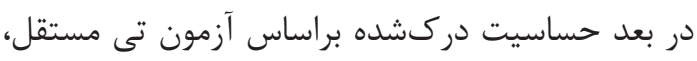

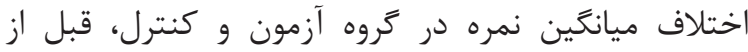

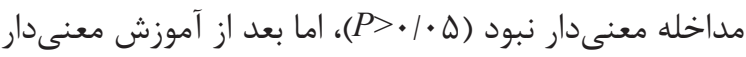

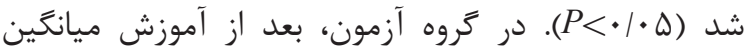

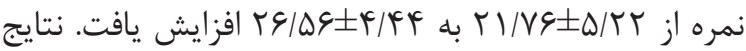

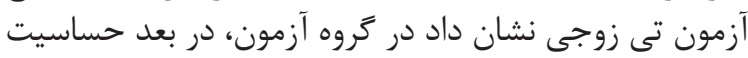

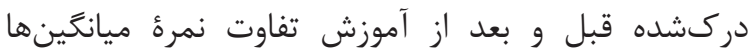

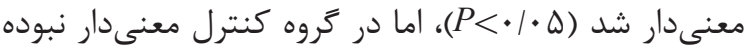

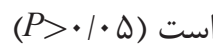

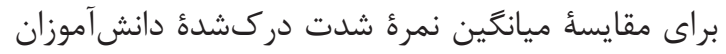

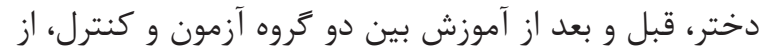

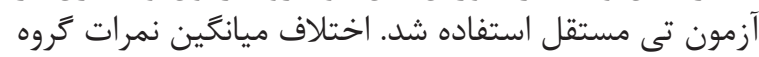

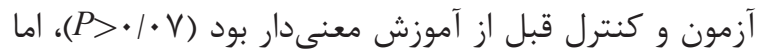

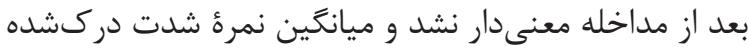
در كروه آزمون از

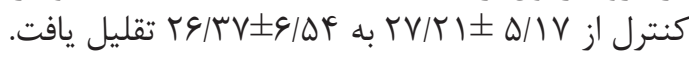

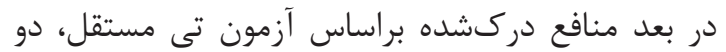

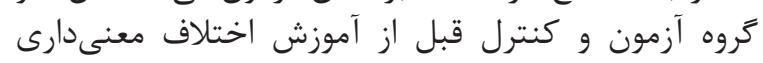

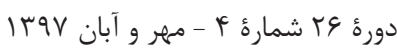

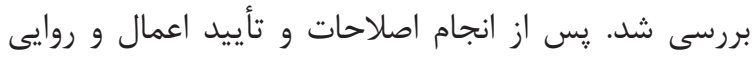

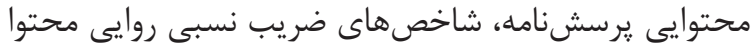

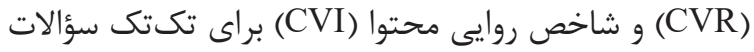

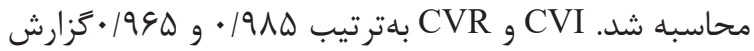
شد كه نشاندهندة اعتبار محتوايى زيادى است

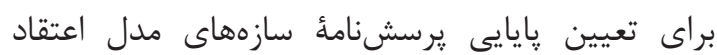

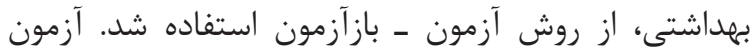

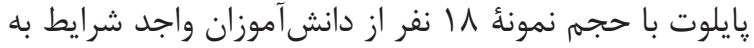

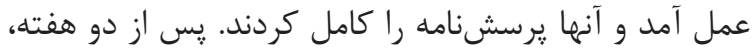

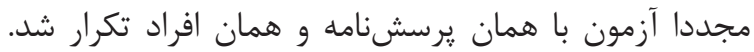

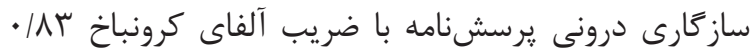

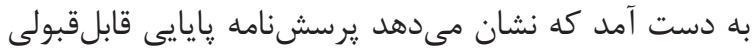

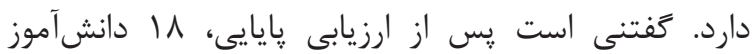

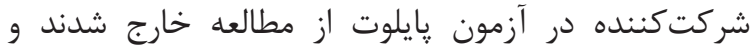

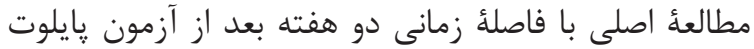

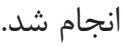

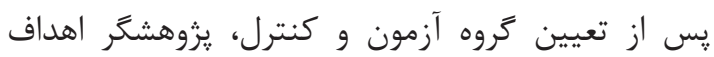

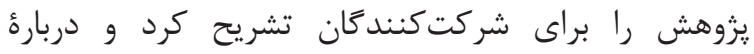

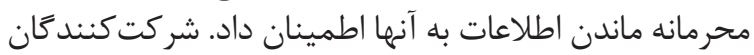

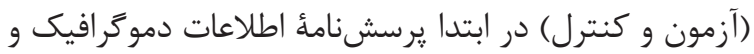

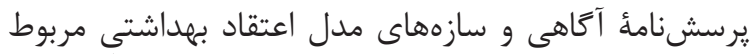

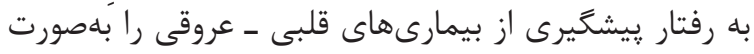

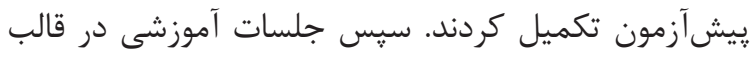

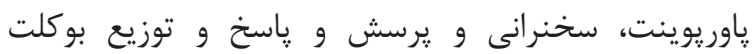

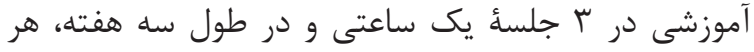

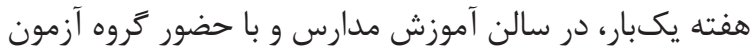

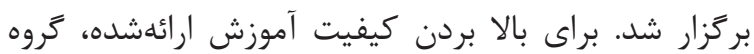

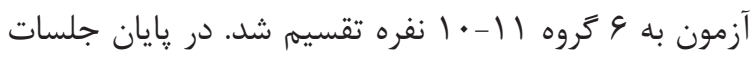

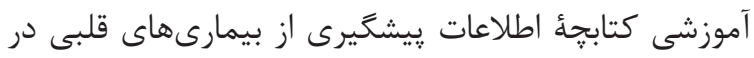

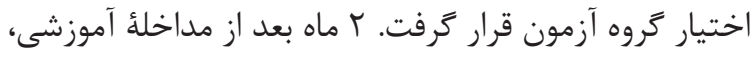

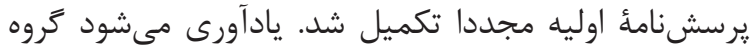

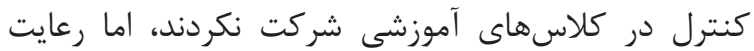

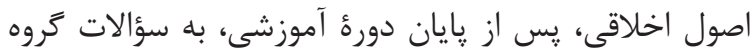

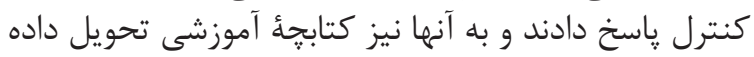

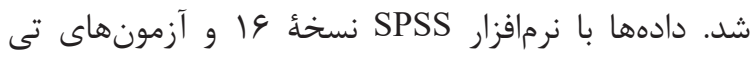

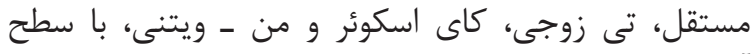

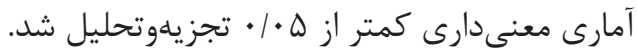

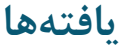

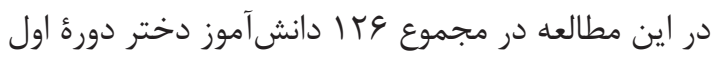

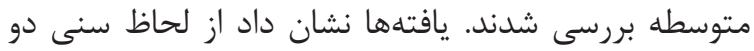

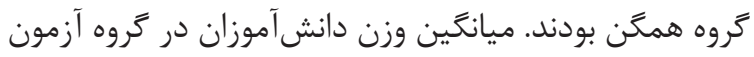

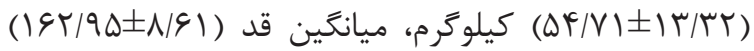

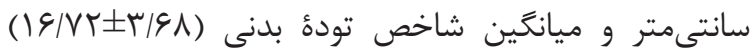

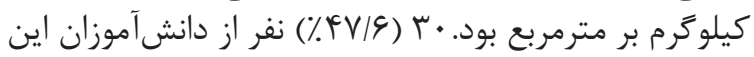

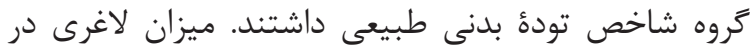

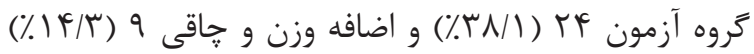

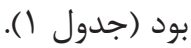

آ 


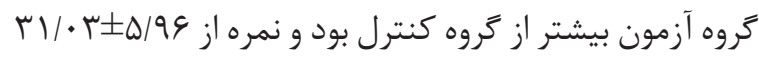

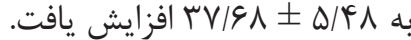

با استفاده از آزمون تى مستقل اختلاف ميانگين نمرة

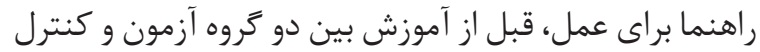
معنى دار بود (T/F)

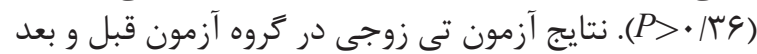

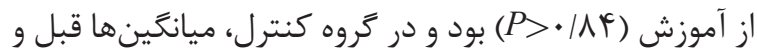

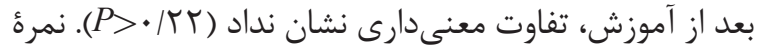

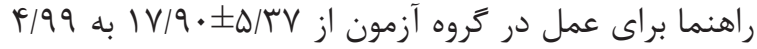

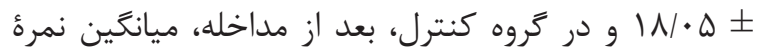

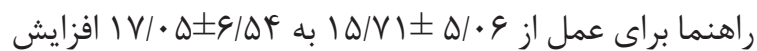

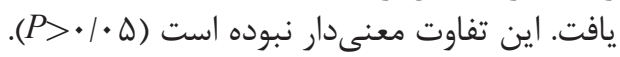

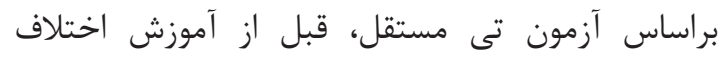

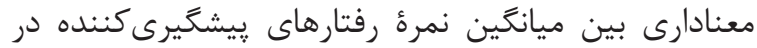

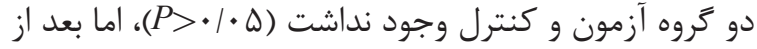

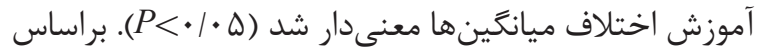

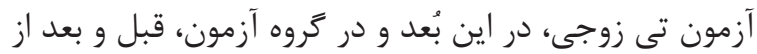

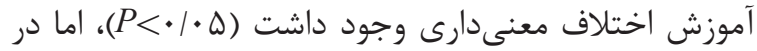

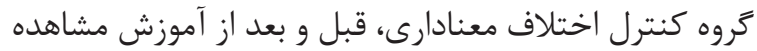

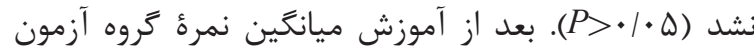

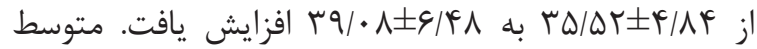

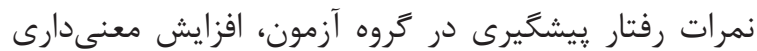

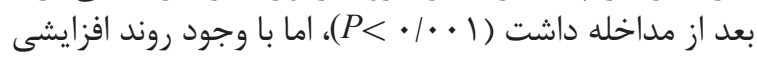

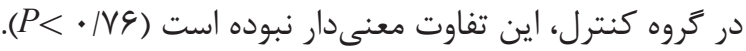

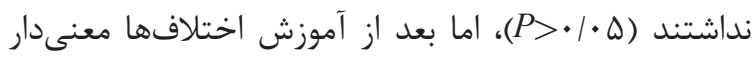

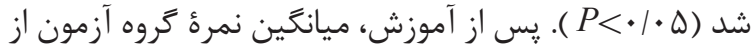
إز

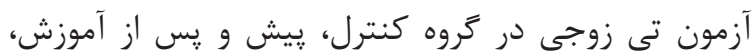

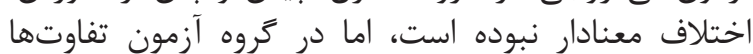

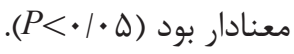

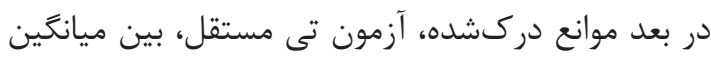

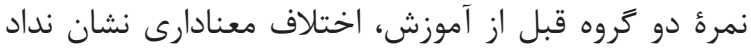

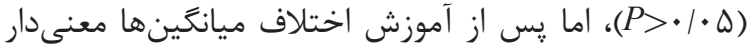

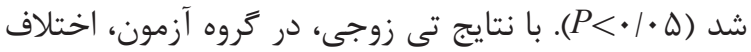

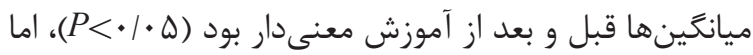

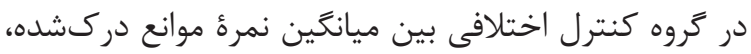

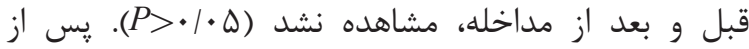

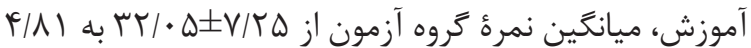

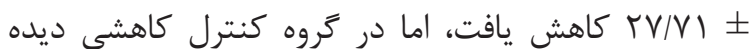

براى مقايسٔ ميانكين نمرهٔ خودكارآمدى دركشده،

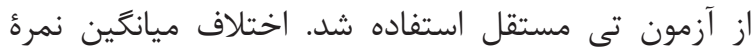

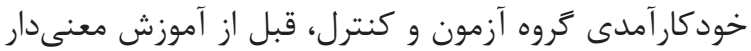

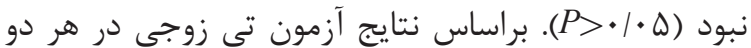

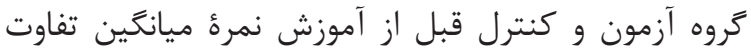

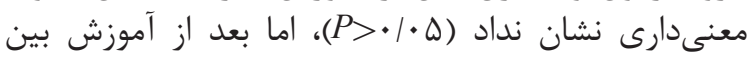

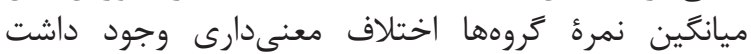

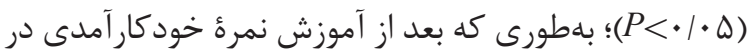

جدول شماره ا. توزيع متغيرهاى دموكر افيك در دانش آموزان به تفكيك تروه آزمون و كنترل

\begin{tabular}{|c|c|c|c|c|}
\hline آزمون آمارى & تعداد (درصد) & 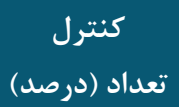 & تروه & متغير \\
\hline \multirow{4}{*}{$\begin{array}{l}\mathrm{X}^{r}=r / 1 \wedge \\
P=\cdot / / r q\end{array}$} & $(I F / T \Lambda) q$ & $(\mid V / F \varepsilon) \mid 1$ & دوازده ساله & \multirow{4}{*}{ سن } \\
\hline & וץ (נז/זr) & $(\Gamma / V Y) Y$. & سيزده ساله & \\
\hline & ו & $(r \cdot / 10) 19$ & קهارده ساله & \\
\hline & $(19 / \cdot F) \mid r$ & $(r \cdot \mid \varepsilon T) \mid r$ & ֶانزده ساله & \\
\hline \multirow{3}{*}{$\begin{array}{l}X^{r}=r / \mu r \\
P=\cdot / 19\end{array}$} & $(r N / I) T F$ & $(\xi 1 / \Gamma) \Gamma \varepsilon$ & لاغر & \multirow{3}{*}{ BMI } \\
\hline & $(F \vee / G) r$. & $(\Delta F / I) T F$ & طبيعى & \\
\hline & $(\mid F / T) q$ & $(\mathcal{F} / \Lambda) \Gamma$ & جاق & \\
\hline \multirow{4}{*}{$\begin{array}{l}\mathrm{X}^{r}=\cdot / \wedge r \\
P=\cdot / \wedge r\end{array}$} & $(I T / I Y) \Lambda$ & $(I T / / Y) \Lambda$ & سه نفره & \multirow{4}{*}{ بعد خانوار } \\
\hline & $(G T / \mid T) F \mid$ & $(\Delta \vee / \Delta) \Psi \wedge$ & جهار نفره & \\
\hline & ru) & $(r \Delta / 9) \backslash \vee$ & ينج نفره & \\
\hline & $(9 / .9)^{f}$ & $(\boldsymbol{\varphi} / \varepsilon) \mu$ & شش نفره & \\
\hline \multirow{3}{*}{$\begin{array}{l}X^{r}=r / \vee \backslash \\
P=\cdot / \wedge r \Delta\end{array}$} & $(19 / 9) 11$ & $(\mid \varepsilon / 9) \mid 1$ & زير دييلم & \multirow{3}{*}{ تحصيلات يدر } \\
\hline & $($ (rq/V)Y I & $($ ( $/ / \Gamma) r \varepsilon$ & دييلم & \\
\hline & $(\mathcal{G} / \mathcal{F}) \mu \mathcal{F}$ & $(F+/) r q$ & بالاتر از دييلم & \\
\hline
\end{tabular}




\begin{tabular}{|c|c|c|c|c|}
\hline آزمون آمارى & تعداد (درصد) & تعداد (درصد) & تروه & متغير \\
\hline \multirow{3}{*}{$\begin{array}{l}X^{r}=r / \Lambda r \\
P=\cdot / \Delta V r\end{array}$} & $(Y T / V) \backslash D$ & $(Y T / V) \backslash \Delta$ & زير دييلم & \multirow{3}{*}{ تحصيلات مادر } \\
\hline & $(F T / F T) Y \Lambda$ & $(q \cdot / 9) r v$ & دييلم & \\
\hline & r & F T (Uس/G) & بالاتر از ديِلم & \\
\hline \multirow{2}{*}{$\begin{array}{l}P=\cdot / \mathrm{r} \cdot \mathrm{r} \\
\mathrm{X}^{r}=\mathrm{V} / 99\end{array}$} & $(\mid r / \varepsilon /)^{9}$ & $(Y T / V) \backslash \Delta$ & شاغل & \multirow{2}{*}{ شغل مادر } \\
\hline & $(\Lambda G / \Gamma) \Delta \vee$ & $(V V / T) D I$ & غيرشاغل & \\
\hline$X^{r}=r / 9 r$ & ( & $(r q / F) r r$ & دولتى & \multirow{2}{*}{ شغل پدر } \\
\hline$P=\cdot / 1 \cdot \varphi$ & $(\Delta \cdot / \wedge \Delta) r r$ & $(\varepsilon \Delta / \varepsilon \cdot) \varphi^{\prime} \mid$ & غيردولتى & \\
\hline
\end{tabular}

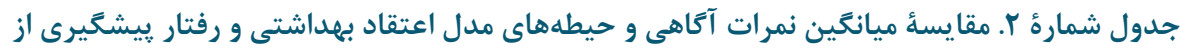

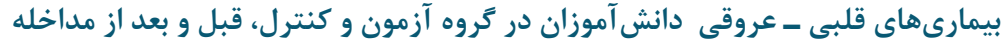

متغير

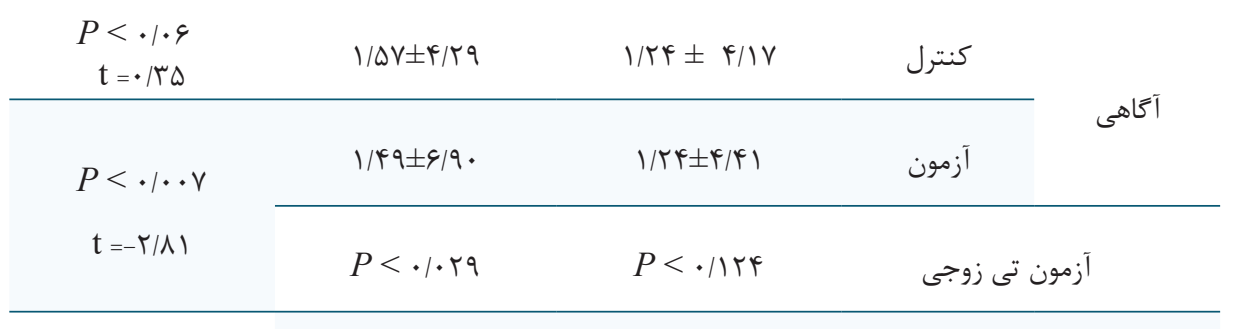

\begin{tabular}{|c|c|c|c|c|}
\hline $\begin{array}{c}P<\cdot / r q \mid \\
\mathrm{t}=r / \mid \vee r\end{array}$ & $r / \cdot \Delta \pm r) / q$. & $r / V I \pm r \mid / r r$ & كنترل & حساسيت \\
\hline$P<\cdot / \cdot r q$ & $\cdot / V Q \pm r \& / \Delta \varphi$ & $\Delta / r T \pm r \mid / V G$ & آزمون & در كشده \\
\hline $\mathrm{t}=-\cdot / \wedge \varphi$ & $P<\cdot / \cdot r \varphi$ & $P<\cdot / r \wedge 1$ & \multicolumn{2}{|c|}{ آزمون تى زوجى } \\
\hline $\begin{array}{c}P<\cdot / \text { FF } \\
\mathrm{t}=1 / 1 \Lambda\end{array}$ & $\varepsilon / D Y \pm r \varepsilon / r V$ & $\Delta / I V \pm r V / r \mid$ & كنترل & \multirow{2}{*}{ دركشده } \\
\hline$P<\cdot / \Delta \mathrm{V}$ & $r q / \cdot r \pm F / T r$ & $r 9 / D 1 \pm 9 / 11$ & آزمون & \\
\hline $\mathrm{t}=\cdot / \Delta \vee \Delta$ & $P<\cdot / T V$ & $P<\cdot / \cdot V$ & \multicolumn{2}{|c|}{ آزمون تى زوجى } \\
\hline
\end{tabular}

\begin{tabular}{|c|c|c|c|c|}
\hline $\begin{array}{l}P<\cdot / q \mu \\
\mathrm{t}=-\cdot / 4 q\end{array}$ & $r q / .9 \pm r / \Delta q$ & $r \Delta / r \Delta \pm r$ & كنترل & منافع \\
\hline$P<\cdot / \cdots 1$ & 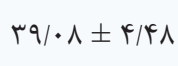 & $r \Delta / \Delta r \pm r / \Lambda F$ & آزمون & 0 \\
\hline $\mathrm{t}=\mathrm{r} / / \mathrm{r}$ & $P<\cdot / \cdot r$ & $P<\cdot / V \mu F$ & \multicolumn{2}{|c|}{ آزمون تى زوجى } \\
\hline $\begin{array}{l}P=\cdot / T r \\
\mathrm{t}=I / T Y\end{array}$ & $r \Lambda / r \Lambda \pm \Lambda / r q$ & $r q / V G \pm \Delta / F r$ & كنترل & \multirow{2}{*}{ موانع دركشده } \\
\hline$P<\cdot / \cdot 1$ & $r V / V I \pm r / \Lambda)$ & $r r / \cdot \Delta \pm V / T \Delta$ & آزمون & \\
\hline $\mathrm{t}=-\mathrm{F} / 99$ & $P<\cdot / \cdots 1$ & $P<\cdot / \wedge$ & \multicolumn{2}{|c|}{ آزمون تى زوجى } \\
\hline
\end{tabular}




\begin{tabular}{|c|c|c|c|c|}
\hline \multirow{2}{*}{ آزمون آمارى } & \multicolumn{2}{|c|}{ انحرافمعيار 土 ميانكين } & \multirow{2}{*}{ تروه - ت ك } & \multirow{2}{*}{ متغير } \\
\hline & دو ماه بعد از مداخله & قبل از مداخله & & \\
\hline $\begin{array}{r}P<\cdot / \Lambda F \\
\mathrm{t}=-1 / K T\end{array}$ & $\mid V / \cdot \Delta \pm \Lambda / q$ & $|\Delta / V| \pm \Delta / \cdot \varphi$ & كنترل & \multirow{2}{*}{ راهنما براى } \\
\hline$P<\cdot /$ A $F$ & $|N| \cdot \Delta \pm r / 99$ & $I V / q \cdot \pm \Delta / T V$ & آزمون & \\
\hline $\mathrm{t}=-\cdot / \mathrm{r} \cdot 1$ & $P<\cdot /$ \& & $P<\cdot / \cdot r$ & \multicolumn{2}{|c|}{ آزمون تى زوجى } \\
\hline $\begin{array}{c}P<\cdot / \mathrm{V} \wedge \\
\mathrm{t}=-1\end{array}$ & $T r / \cdot r \pm \Delta / T r$ & $r / / V \pm \Delta / r T$ & كنترل & \multirow{2}{*}{ خود كار آمدى } \\
\hline$P<\cdot / \cdot \vee V$ & $r V / G \Lambda \pm \Delta / F V$ & $r / / r \pm \Delta / ৭ \varphi$ & آز آزمون & \\
\hline$t=-r / \cdot r$ & $P<\cdot / \cdot r F$ & $P<\cdot / r q 4$ & \multicolumn{2}{|c|}{ آزمون تى زوجى } \\
\hline $\begin{array}{c}P<\cdot / V G \\
\mathrm{t}=\cdot / \Gamma /\end{array}$ & $r / / 1 \pm r / 9 r$ & $r \cdot / 9 \Lambda \pm r / \Lambda 1$ & كنترل & \multirow{2}{*}{ ييشخيرى } \\
\hline$P<\cdot / \cdot 1$ & $r \&|\Delta| \pm 1 / 9 V$ & rI/VA \pm r/lৎ & آزمون & \\
\hline $\mathrm{t}=-\varepsilon / \Delta T$ & $P<\cdot / \cdot 1$ & $P<\cdot / \pi \mid r$ & \multicolumn{2}{|c|}{ آزمون تى زوجى } \\
\hline
\end{tabular}

بيشَيرى كننده از بيمارىهاى قلبى - عروقى، در كروه

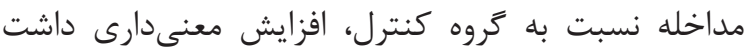

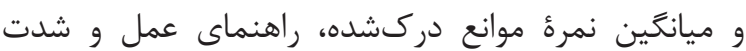
دركشده كاهش معنى دارى رانع در نشان داد.

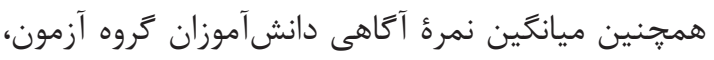

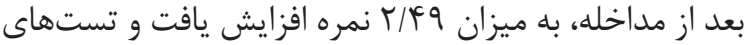

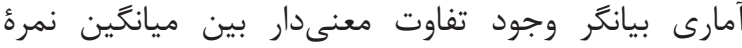

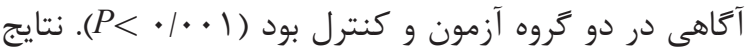

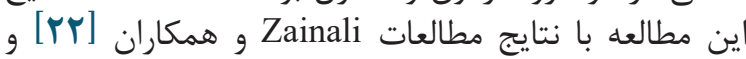

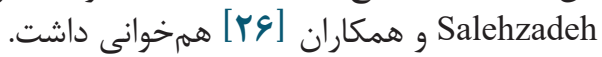

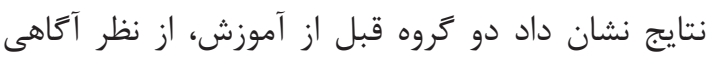

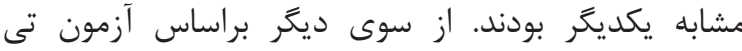

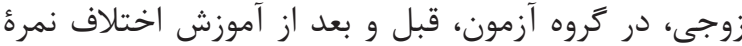

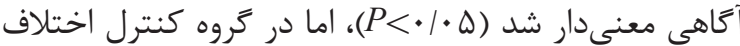

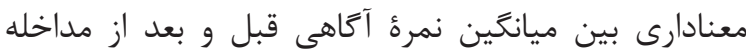

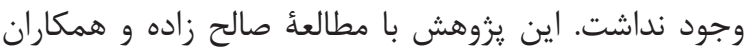

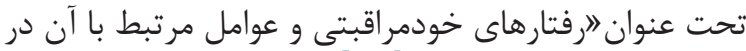

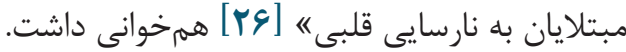

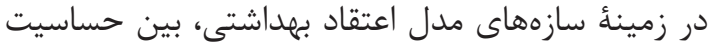

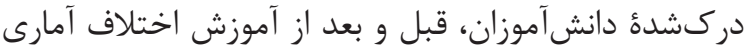

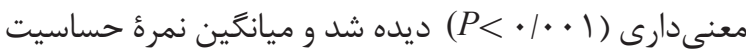

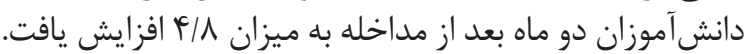

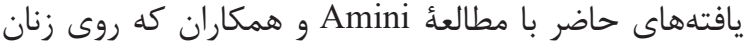

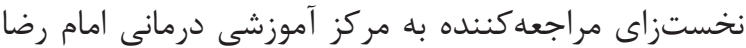

\section{بحث}

يثرهش حاضر با هدف تعيين تأثير آموزش براساس مدل

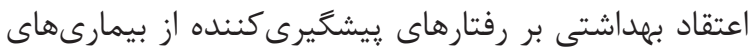

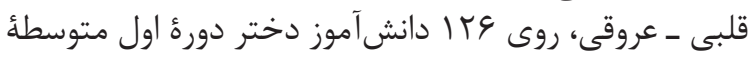

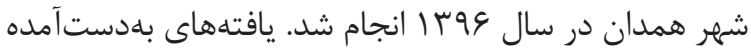

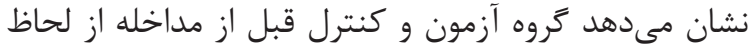

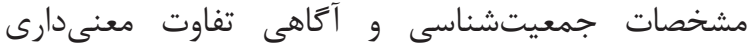
نداشتند و همخًن بودند (ه • • • (P) )

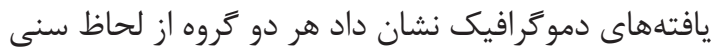

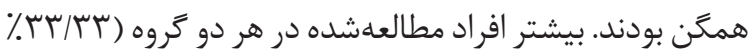

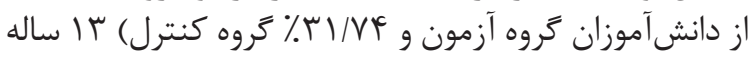

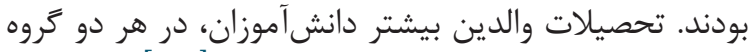

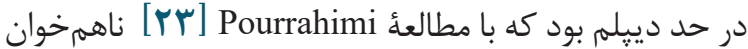

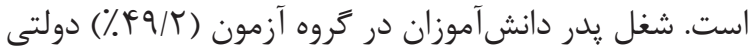

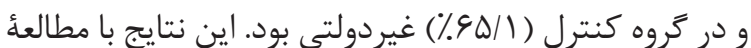

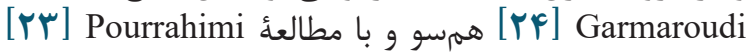

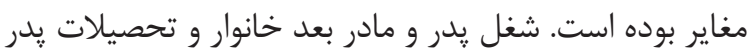

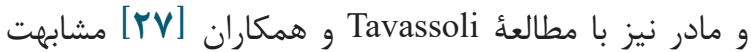

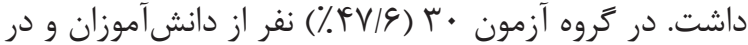

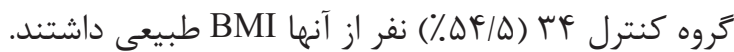

نتايج تحليل سازههاى مدل اعتقاد بهداشتى در زمينأ

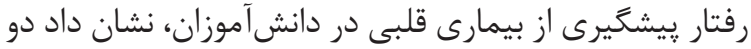

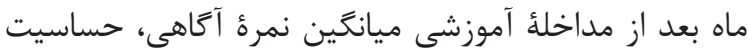

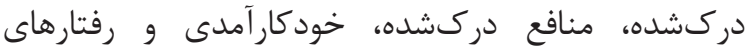

$$
\text { مجلئ علمى دانشكدة يرستارى و مامايى همدان }
$$


وجود نداشت و بعد از مداخله نيز اختلاف معنادارى بين دو

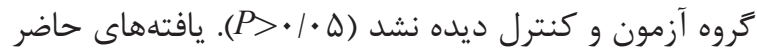

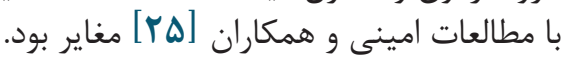

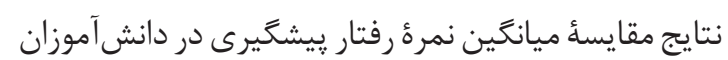

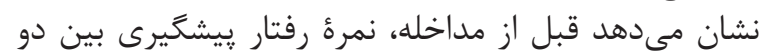

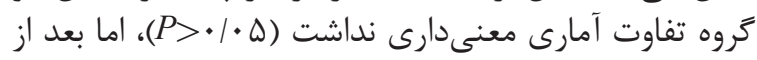

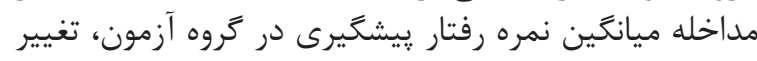

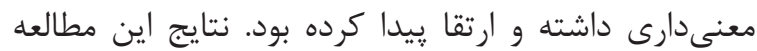

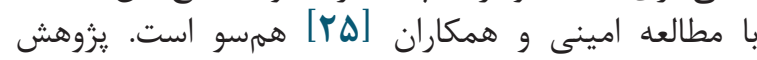
حاضر درباره وضعيت عملكرد دانشآموزان درباره

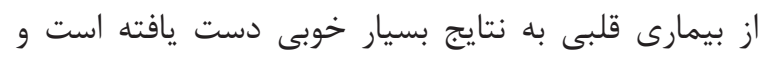

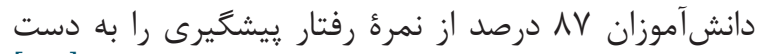

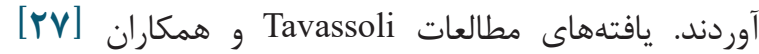

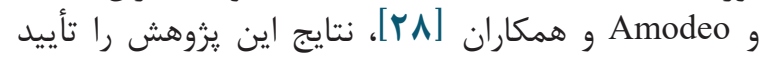

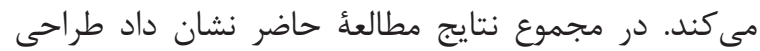

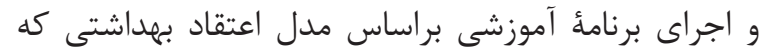

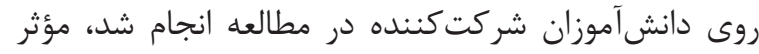
بوده است.

\section{نتيجهة تيرى}

نتايج اين مطالعه حاكى از آن است كه آموزش مبتنى بر

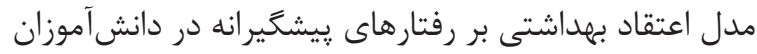

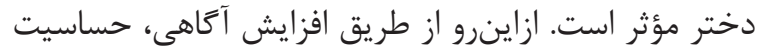

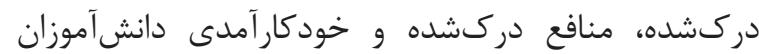

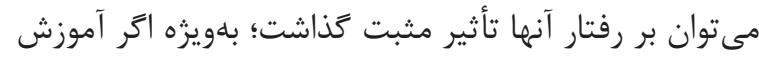
از سنين يايينتر شروع شود.

\section{سياسگزارى}

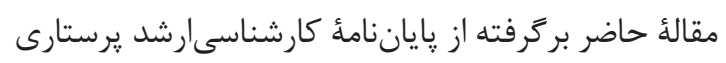

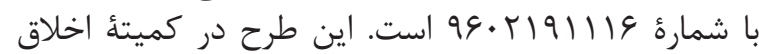

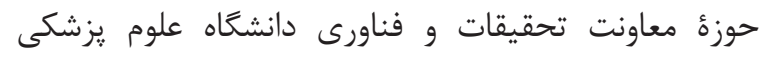

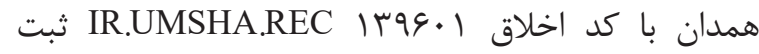

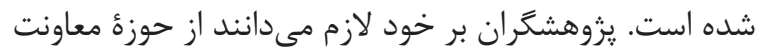

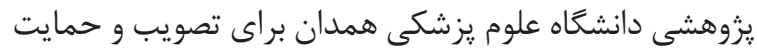

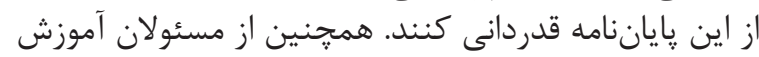

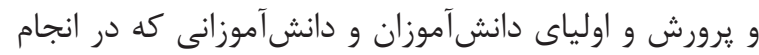

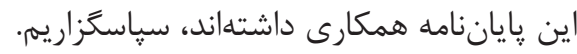

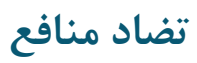

بين نويسند أن هيجگَونه تضاد منافعى وجود ندارد.

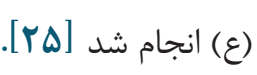

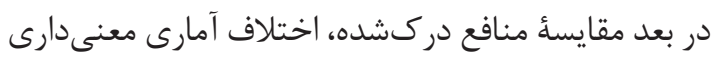

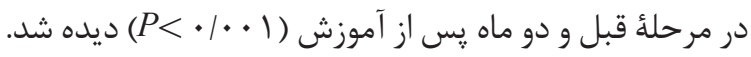

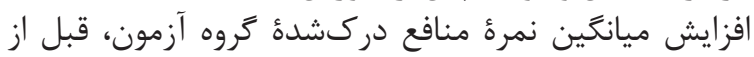
مداخله

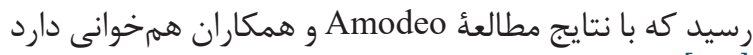

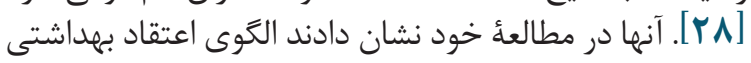

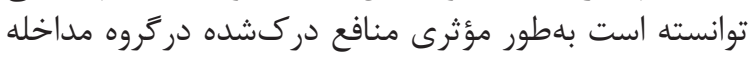

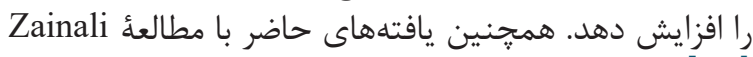

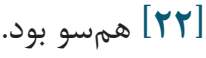
در بعد شدت دركشده اختلاف آمارى معنى مدارى،

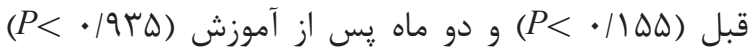

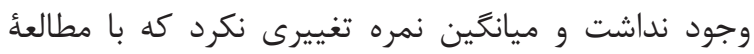
Mansourian

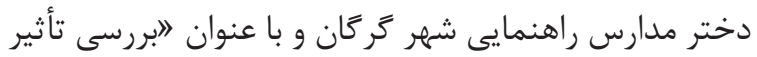

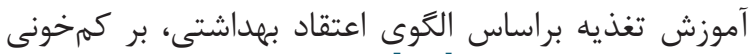

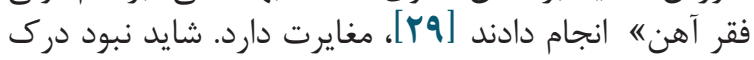

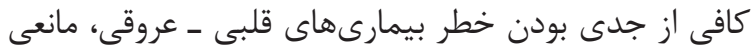

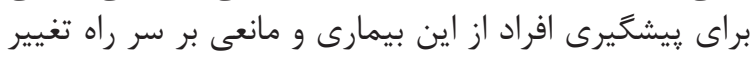
سبك زند سئى در آنها باشد.

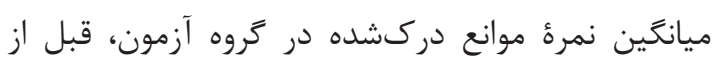

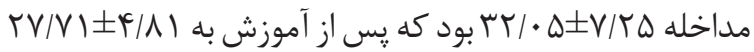

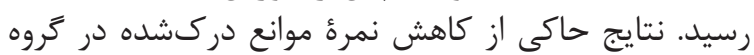

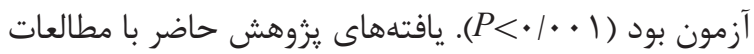
Amini

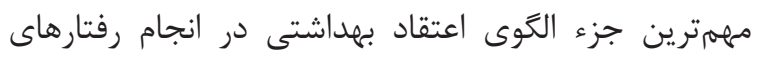
ييشخَير انه است.

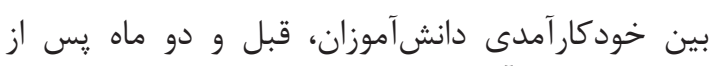

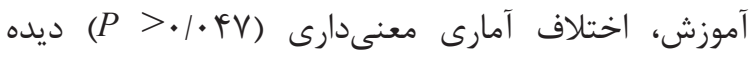

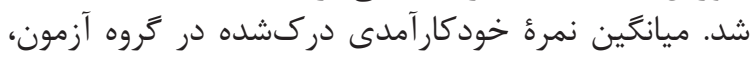

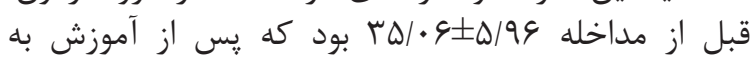

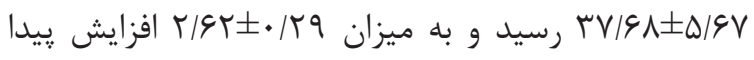

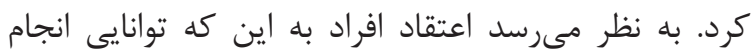

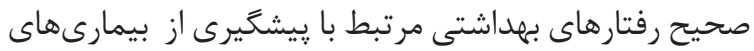

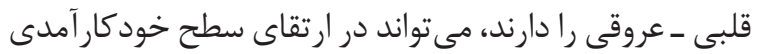

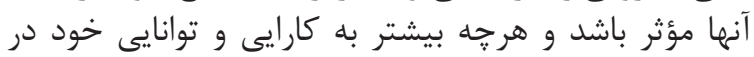

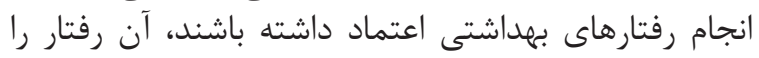

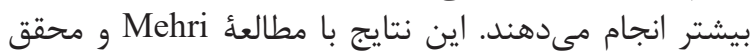

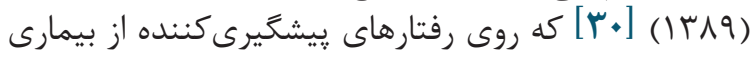

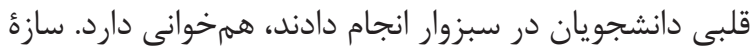

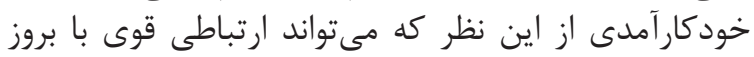

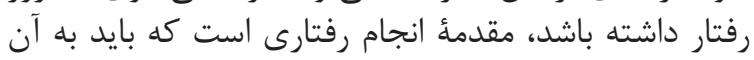
توجه كرد.

در بعد راهنما براى عمل، نتايج نشان داد اختلاف آمارى

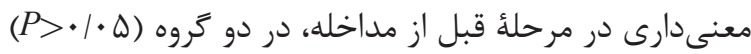


ناهيد محمدى و همكاران. هسr

\section{References}

1. Moshki M, Mojadam M, Dusti Irani A. Associated Factors for Preventive Behaviors of Cardiovascular Diseases in Employees of Khuzestan Province Health Center Utilizing the Health Belief Model. j.health. 2015; 6 (4) :367-377 http:// healthjournal.arums.ac.ir/article-1-746-en.html

2. Poudel K, Sumi N. Health Behavior Regarding Cardiovascular Diseases Among Nepali Adults. J Community Health. 2017;42(6):1240-6. PMid:28523570

3. Imanipour M, Bassampour S, Haghani H. Relationship between preventive behaviors and knowledge regarding cardiovascular diseases. Journal of hayat. 2008;14(2):41-9.

4. Chateau-Degat ML, Dewailly É, Louchini R, Counil É, Noël M, Ferland A, Lucas M, Ékoé JM, Ladouceur R, Déry S, Egeland GM. Cardiovascular burden and related risk factors among Nunavik (Quebec) Inuit: insights from baseline findings in the circumpolar Inuit health in transition cohort study. Canadian Journal of Cardiology. 2010; 26(6):e190-6. https://doi.org/10.1016/ $\underline{\mathrm{S} 0828-282 X(10) 70398-6}$

5. Azizi F, Emami H, Salehi P, Ghanbarian A, Mirmiran P, Mirbolooki M. Cardiovascular risk factors in the elderly: Tehran Lipid and Glucose Study (TLGS). Iranian Journal of Endocrinology and Metabolism. 2003; 5 (1):3-13

6. Oliver-Mcneil S, Artinian NT. Women's perceptions of personal cardiovascular risk and their risk-reducing behaviors. Am J Crit Care. 2002;11(3):221-7 .PMid:12022485

7. Varo JJ, Martinez-Gonzalez MA, De Irala-Estevez J, Kearney J, Gibney M, Martinez JA. Distribution and determinants of sedentary lifestyles in the European Union. Int J Epidemiol. 2003;32(1):138-46. https://doi.org/10.1093/ije/ dyg116 PMID: 12690026

8. Barratt J. Diet-related knowledge, beliefs and actions of health professionals compared with the general population: an investigation in a community Trust. J Hum Nutr Diet. 2001;14(1):25-32. https://doi.org/10.1046/ j.1365-277X.2001.00267.x PMID: 11301929

9. Mackay J. The atlas of heart disease and stroke. World Health Organization; 2004.

10. Hosseinnezhad M. Azizadeh Foorouzei M, Mohammadalizadeh S, Haghdoust A. Assessment of the self efficacy role in prediction of nutritional behavior of girls students. Journal of Shahid Sadooghi University of Medical Sciences of Yazd. 2008;16:49-56.

11. Rezaiepour A, Yousephei F, Mahmoodi M, Shak- eri M. the relationship of nutritional behaviors and physical Activities of adolescent girls with their perception of parental lifestyle. Journal of Nursing and Midewifery College, Tehran University of Medical Sciences (Hayat). 2007;13:17-25.

12. Pirasteh A, Heidarniya A, Faghiehzadeh S. Build and validate scales measuring psychosocial Factors. Which are effective in physical activity of high school girls of district 10 of Tehran. Journal of Ilam University of Medical Sciences. 2007; 15:39-47.

13. https://www.amar.org.ir/

14. Ahadi Z, Shafiee G, Qorbani M, Sajedinejad S, Kelishadi R, Arzaghi SM, et al. An overview on the successes, challenges and future perspective of a national school-based surveillance program: the CASPIAN study. Journal of Diabetes \& Metabolic Disorders. 2014;13(1):120. https://doi.org/10.1186/s40200-014-0120-3 PMID:25614853 PMCid:PMC4302132

15. Long JD, Stevens KR. Using Technology to Promote Self Efficacy for Healthy Eating in Adolescents. Journal of Nursing Scholarship. 2004;36(2):134-9.https://doi.org/10.1111/j.15475069.2004.04026.x PMID: 15227760

16. Speizer IS, Tambashe BO, Tegang SP. An evaluation of the "Entre nous jeunes" peer-educator program for adolescents in Cameroon. Studies in family planning. 2001;32(4):339-51. https:// doi.org/10.1111/j.1728-4465.2001.00339.x PMID: 11831052

17. Okada M, Tsunoda M, Satoh T, Aizawa Y. A lifestyle to prevent or combat the metabolic syndrome among Japanese workers: analyses using the health belief model and the multidimensional health locus of control. Industrial health. 2011;49(3):365-73. https://doi.org/10.2486/indhealth.MS1172 PMID:21372436

18. Kazemi A, Ehsanpour S, Nekoei-Zahraei NS. A randomized trial to promote health belief and to reduce environmental tobacco smoke exposure in pregnant women. Health education research. 2012;27(1):151-9. https://doi.org/10.1093/her/ cyr102 PMID:22052216

19. Heidarnia A. Discussion in health education process: Zamani publication; 2003: 91-4.

20. Khosravi S, Jahani F, Gazerani N, Eshrati B, Moghimi M. Determining knowledge and attitude of students in Arak University of medical sciences about Ecstasy based on Health Belief Model in 2006. Arak Medical University Journal. 2007;10(2):15-21.

21. Burbank PM, Riebe D. Promoting exercise and behavior change in older adults: interventions with the transtheoretical model: Springer Pub- 
lishing Company; 2001.

22. Zainali M, Asadpour M, Aghamolaei T, Esmaeili Nadimi A, Farshidi H, Ghanbarnejad A. Effect of educational intervention based on health belief model to promote preventive behaviors of cardiovascular disease in people with normal angiographic results. Journal of Preventive Medicine. 2015;1(2):1-12.

23. Pourrahimi M, Yahyavi SH, Safavi M, Mahmoudi M. The Effect of Education on the Self-efficacy of Nutritional Behaviors of Secondary School Students in Kerman city. Medical Science Journal of Islamic Azad Univesity-Tehran Medical Branch. 2012;22(2):143-51.

24. Garmaroudi G, Sarlak B, Rahimi Foroushani A. The Effect of an Intervention Based on the Health Belief Model on Preventive Behaviors of Domestic Violence in Female High School Students. Journal of Knowledge \& Health Vol 11, No 1Shahroud University of Medical Sciences. 2016;11:69-73.

25. Amini R, Moayeri E, Khodaveis M. The Effect of Health Belief Model Based Education on Breastfeeding of Unipara Mothers Referred to Imam Reza Educational Hospital. Iranian Journal of Pediatric Nursing (JPEN). 2016;3(2).

26. Salehzadeh A, Rahmatpour P. Self-care behav-

$$
\text { צYr. تأثير آموزش براساس مدل اعتقاد بهداشتى ... }
$$

iors and related factors in patients with heart failure reffering to medical \& educational center of heart in Rasht.. Journal of Holistic Nursing And Midwifery. 2013;23(1):22-9.

27. Tavassoli E, Reisi M, Javadzade H, Mazaheri M, Ghasemi S, Shakoori S. The effect of the health belief model-based education \& improvement of consumption of fruits and vegetables: An interventional study. Journal of Health in the Field. 2017;1(2).29-35

28. Amodeo R, A DP, Sorbara L, Avanzini F. How to increase patient knowledge of their coronary heart disease: impact of an educational meeting led by nurses.G Ital Cardiol. 2009; 10(4):: 256.8 .

29. Mansourian M, shafieyan Z, Qorbani M, Rahimzadeh Bazraki H, Charkazi A, Asayesh $\mathrm{H}$, et al . Effect of nutritional education based on HBM model on anemia in Golestan girl guidance school students. Iran J Health Educ Health Promot. 2013; 1 (2) :51-56.

30. Mehri A, Mohaghegh NM. Utilizing the health belief model to predict preventive behaviors for heart diseases in the students of Islamic Azad university of Sabzevar (2010). Toloo-Behdasht. 2010;9(2-3):21-33. 